\title{
The price of anarchy for utilitarian scheduling games on related machines
}

\author{
Ruben Hoeksma ${ }^{\mathrm{a}}$, Marc Uetz ${ }^{\mathrm{b}}$ \\ ${ }^{a}$ Center for Mathematical Modeling, University of Chile, Beaucheff 851, Santiago, Chile \\ ${ }^{b}$ Dept. Applied Mathematics, University of Twente, P.O. Box 217, 7500AE Enschede, The \\ Netherlands
}

\begin{abstract}
We present bounds on the efficiency of Nash equilibria in a scheduling game where jobs are players who choose a machine out of a set of machines to be processed on. Machines may have different speeds, and sequence the jobs in shortest processing time first order. When players selfishly choose a machine to minimize their own completion time, we analyze the price of anarchy for the sum of the completion times of the jobs. We show that it is bounded from below by $e /(e-1) \approx 1.58$ and from above by 2 .
\end{abstract}

Keywords: price of anarchy, scheduling, utilitarian, related machines

\section{Introduction}

In settings with multiple noncooperative decision makers, it is well known that the lack of central coordination may cause a loss in overall efficiency. The systematic study of what is now known as the price of anarchy [22] was initiated in a 1999 paper by Koutsoupias and Papadimitriou [19], even though there was earlier work to address the inefficiency of equilibria, e.g., Dubey [11]. Koutsoupias and Papadimitriou, however, suggested to systematically compare the worst case social cost of a solution that may arise as a Nash equilibrium to that of a solution with minimum social cost. Since then, the price of anarchy has been analyzed in many game theoretic versions of combinatorial optimization problems, most prominently auctions, network routing and scheduling. Specifically, the literature on analysis of the price of anarchy for scheduling problems is very extensive. Various models exist in which either machines or jobs are interpreted as selfish agents, and different objectives may be studied that depend on either machine loads $L_{i}$ or job completion times $C_{j}$. Most notably, following Koutsoupias and Papadimitriou [19], a lot of work was done on the egalitarian ${ }^{1}$ makespan, $C_{\max }\left(=\max _{j} C_{j}\right)$, as social cost function [3, 6, 10, 18, 19, 26. Indeed, the interpretation of the makespan objective as egalitarian makes sense in models where the objectives of the job-agents is the total load $L_{i}$ of the machine $i$ they are processed on, as for example in Koutsoupias and Papadimitriou [19]. Later, publications have also addressed models in which job-agents

\footnotetext{
Email addresses: rhoeksma@dim.uchile.cl (Ruben Hoeksma), m.uetz@utwente.nl (Marc Uetz)

${ }^{1}$ See Myerson [21] for a discussion of utilitarian and egalitarian social choice functions. 
$j$ care about their own completion time $C_{j}$ in a schedule, hence the order of jobs per machine plays a role [4, 7, 9, 23, and the utilitarian social cost function is then $\sum_{j} C_{j}$, or $\sum_{j} w_{j} C_{j}$ with individual job weights $w_{j}$.

This paper addresses one of the simplest, non-trivial utilitarian machine scheduling games: Job-agents select a machine to be processed on, with the goal to minimize their individual completion times $C_{j}$. The utilitarian social cost function is then $\sum_{j} C_{j}$. In order to define the game that is being played by the jobs, we make the natural assumption that each machine sequences its jobs in the locally optimal order for the objective $\sum_{j} C_{j}$, which is shortest processing time first (SPT).

The main result in this paper is an analysis of the price of anarchy when the machines may have different speeds. That model is denoted $\mathrm{Q} \| \sum C_{j}$ in the three-field scheduling notation of Graham et al. [13. We also briefly comment on the case where all these machine speeds are identical, known as $\mathrm{P} \| \sum C_{j}$. More specifically, we show that the price of anarchy for the case where machines may have different speeds is at most 2 and at least $e /(e-1) \approx 1.58$. When all speeds are identical, the price of anarchy equals $3 / 2-1 /(2 m)$, where $m$ is the number of machines. The latter result appears to be a bit of folklore in the community; it shows up as a special case, e.g. in [23. We include it with a simple proof for the sake of completeness.

We believe that our results are interesting for mainly two reasons. First, an interesting feature of the problem we address is the fact that both, computation of a (pure) Nash equilibrium and an optimal solution can be done in polynomial time by simple, well known algorithms. In fact, for the problem we address it is well known that (pure) Nash equilibria are obtained as solutions of the Ibarra and Kim algorithm [17. Moreover, an optimal solution is obtained by the Minimum Mean Flow Time (MFT) algorithm by Horowitz and Sahni [16. Yet, despite this fact which seems to place the problem that we address here close to trivial, we require a new characterization of optimal solutions to get our analysis done. That characterization is interesting in its own right. Moreover, despite being a target in the community since several years, since the publication of the conference paper where our results have been announced [15, the gap for the price of anarchy has not been closed and seems to require new techniques.

As to related work, the papers by Correa and Queyranne 9, and Cole et al. 7] are very closely related to our work. Both address the same problem as we do, but with additional job weights and in the more general context of unrelated machine scheduling. One of the main results in both papers is a proof that the price of anarchy is 4 when machines sequence their jobs locally optimal, that is, according to non-increasing ratios of weight over processing time. Cole et al. [7 also give an instance which establishes a lower bound of 4 for the price of anarchy, even in the unweighted case. Our paper adds to this line of work by improved price of anarchy bounds for a special case, obtained by completely different techniques.

The organization of this paper is as follows. In Section 3 we briefly recap the Minimum Mean Flow Time (MFT) algorithm by Horowitz and Sahni [16. We then present a new characterization of optimal solutions, which is crucial for the subsequent analysis. In Section 4.1 we show that the price of anarchy is not greater than 2. The proof goes by showing what is now known as $(2,0)$-semi-smoothness of the game 1, 5. To do that, our new characterization of optimal solutions is key. Section 4.2 then describes a parametric instance, for which we show that its a price of anarchy is (asymptotically) equal to $e /(e-1)>1.58$. We briefly address the special case of identical machines in 
Section 5 and conclude with some remarks in Section 6 ,

\section{Preliminaries}

In this paper we are interested in the price of anarchy in the following, related machine scheduling game. Given are a set $J$ of $n$ jobs and a set $M$ of $m$ machines. Each job $j$ has a processing requirement $p_{j}$ and each machine $i$ has a speed $s_{i}$. The processing time of job $j$ on machine $i$ is equal to $p_{j} / s_{i}$. Without loss of generality we assume that $p_{1} \leq p_{2} \leq \cdots \leq p_{n}$ and $s_{1} \leq s_{2} \leq \cdots \leq s_{m}$. In the case of ties on the ordering, we assume these are broken consistently and that this is done based on index. Each job $j$ is owned by a different strategic player that tries to minimize the completion time $C_{j}$, while the social cost function is utilitarian, meaning that it is the sum of the players' utilities, which is the total completion time of the jobs $\sum C_{j}$. We discuss the setting where on each machine the jobs are processed in shortest processing time first (SPT) order. For this setting pure Nash equilibria always exist. While scheduling the jobs in SPT order is optimal on a local, per machine level, the resulting Nash equilibria are not globally optimal in general. We denote the strategies or choices of the players by a vector $\sigma$ such that $\sigma_{j}$ denotes the machine that job $j$ chooses, or, in the case of mixed strategies, the probability distribution over the machines chosen by job $j$. Moreover, we let $\sigma_{-j}$ denote the $(n-1)$-vector obtained from $\sigma$ by deleting $\sigma_{j}$, such that $\sigma=\left(\sigma_{j}, \sigma_{-j}\right)$. By $C_{j}(\sigma)$ we denote the (expected) completion time of job $j$ given the strategy vector $\sigma$ and by $C(\sigma)=\sum_{j \in J} C_{j}(\sigma)$ we denote the total (expected) completion time corresponding to that same strategy vector $\sigma$.

For games with utilitarian social choice function, Roughgarden 25] introduced the concept of smoothness of games and its consequences for robust price of anarchy bounds. Smoothness of a game directly implies upper bounds on the price of anarchy for pure Nash equilibria that also extends to mixed Nash equilibria, correlated equilibria, and no-regret sequences 25. In contrast to regular smooth games, our analysis for the upper bound crucially needs properties of optimal solutions. It turns out that the conditions for smoothness can be relaxed to allow the use of properties of particular solutions, without losing the generality of the bounds [1, 20, 25]. This relaxed version of smoothness has been called semi-smoothness by Lucier and Paes Leme [20. Anshelevich et al. 1] and Caragiannis et al. [5] give an even more relaxed definition of semi-smoothness that is still lossless in the same sense.

Definition 1 (1, 5] Semi-smooth games). A cost-minimization game is $(\lambda, \mu)$-semismooth if for every player $j$ there exists a mixed strategy $\sigma_{j}$ such that for every strategy vector $\nu$,

$$
\mathbb{E}_{\sigma}\left[\sum_{j=1}^{n} C_{j}\left(\sigma_{j}, \nu_{-j}\right)\right] \leq \lambda \cdot C\left(\sigma^{*}\right)+\mu \cdot C(\nu),
$$

where $\sigma^{*}$ is an optimal solution.

If a utilitarian game is $(\lambda, \mu)$-semi-smooth with $\lambda \geq 0$ and $\mu<1$, it follows that for any (mixed) Nash equilibrium $\nu$ and optimal solution $\sigma^{*}$

$$
\sum_{j=1}^{n} C_{j}(\nu) \leq \sum_{j=1}^{n} C_{j}\left(\sigma_{j}, \nu_{-j}\right) \leq \lambda \cdot C\left(\sigma^{*}\right)+\mu \cdot C(\nu),
$$


where $\sigma_{j}$ is the strategy from (11). It follows directly that $\frac{\lambda}{1-\mu}$ is an upper bound on the price of anarchy for any $(\lambda, \mu)$-semi-smooth game.

\section{Characterization of Optimal Solutions}

In this section we briefly recap the MFT algorithm by Horowitz and Sahni [16] and establish a new characterization for optimal solutions for related machine scheduling. This characterization is crucial to our analysis in Section 4.1 .

When considering only a single machine it is clear that the contribution of a job can be measured by its position in the schedule, and its processing time. This follows from rewriting the objective function as follows. Let $\varphi$ be an ordering of the jobs and let $\varphi(k)$ denote the $k$-th job in this ordering, then the sum of completion times when processing the jobs in that order on a single machine with speed 1 is equal to

$$
\sum_{k=1}^{n} C_{\varphi(k)}=\sum_{k=1}^{n} \sum_{l=1}^{k} p_{\varphi(l)}=\sum_{k=1}^{n}(n-k+1) p_{\varphi(k)} .
$$

Hence, the only optimal schedules are schedules that schedule the jobs in order of nondecreasing processing time, as these match large $p_{j}$ to small values $(n-k+1)$. This idea can be extended to the case of parallel machines, even with speeds, resulting in the MFT algorithm (Algorithm 1) [16, Algorithm 1]. In the MFT algorithm, similar to the single machine case, we make use of the values $\left(z_{i}+1\right) / s_{i}$ which are the values for a job's possible positions in the schedule, as, in general, the $x$-th last job on a machine contributes to the objective value $x$ times its processing requirement divided by the machines speed. The algorithm assigns the currently longest unscheduled job to the machine with the currently smallest position value.

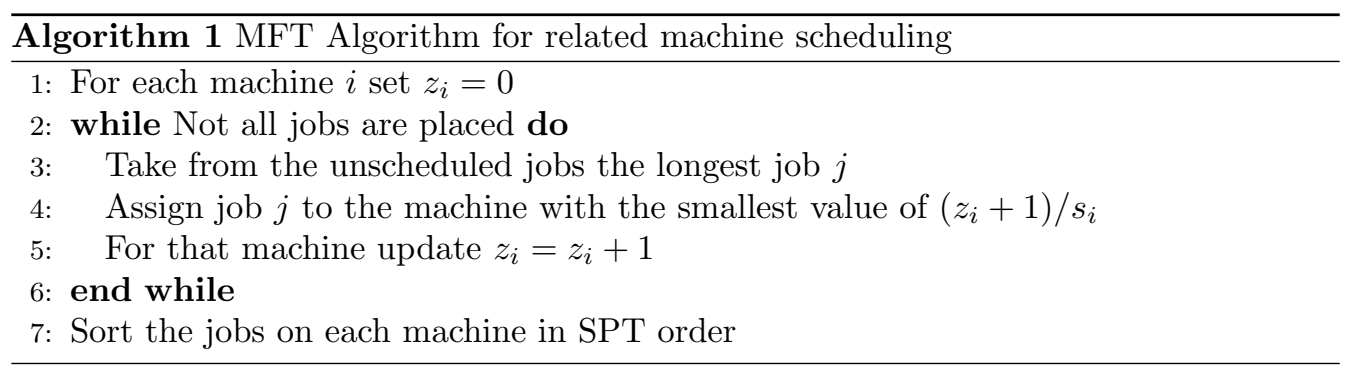

Theorem 1 (Horowitz and Sahni [16]). The MFT algorithm produces optimal schedules for $\mathrm{Q} \| \sum C_{j}$ and any optimal schedule or $\mathrm{Q} \| \sum C_{j}$ can be computed by the MFT algorithm with the proper tie breaking rule.

From here on, for each machine we let the jobs assigned to it be scheduled in SPT order. That said, we identify a schedule with an $n$-vector $\sigma$, where $\sigma_{j}$ is the machine on which job $j$ is scheduled, similar to a strategy vector.

Next, let $z(\sigma, j)$ be the vector such that $z_{i}(\sigma, j)=\left|\left\{k>j \mid \sigma_{k}=i\right\}\right|$. It is the number of jobs on machine $i$ in schedule $\sigma$ that have higher index than $j$. Then, any schedule $\sigma$ 
is optimal if and only if

$$
\frac{z_{\sigma_{j}}(\sigma, j)+1}{s_{\sigma_{j}}} \leq \frac{z_{i}(\sigma, j)+1}{s_{i}}
$$

for all jobs $j$ and all machines $i$. This because, for all machines $i,\left(z_{i}(\sigma, j)+1\right) / s_{i}$ is the value of the next position on machine $i$ upon placement of job $j$ by the MFT algorithm. Indeed, $p_{j}\left(z_{\sigma_{j}}(\sigma, j)+1\right) / s_{\sigma_{j}}$ is exactly the contribution of job $j$ to the objective value in schedule $\sigma$. The sum of these contributions needs to be minimized by any optimal schedule. The following provides our new characterization of optimal solutions; we labelled it as theorem because we think it is interesting in its own right.

Theorem 2. A schedule $\sigma$ is optimal for $\mathrm{Q} \| \sum C_{j}$ if and only if $\mathrm{L}^{2}$

$$
\frac{z_{i}(\sigma, j)+1}{s_{i}} \geq \frac{z_{\ell}(\sigma, j)}{s_{\ell}}
$$

for all machines $i$ and $\ell$.

Proof. We show that (3) is true if and only if (2) is true. Let $\sigma$ be an optimal schedule and let the ordering of the jobs be fixed and in SPT order. Note that $z_{i}(\sigma, j) \geq z_{i}(\sigma, k)$ for all machines $i$ and all jobs $k \geq j$. Therefore, we have from (2) that

$$
\frac{z_{i}(\sigma, j)+1}{s_{i}} \geq \frac{z_{i}(\sigma, k)+1}{s_{i}} \geq \frac{z_{\sigma_{k}}(\sigma, k)+1}{s_{\sigma_{k}}},
$$

for all machines $i$ and all jobs $k \geq j$. Since for any machine $\ell$ either $z_{\ell}(\sigma, j)=0$, or there is a job $k>j$ such that $\sigma_{k}=\ell$ and $z_{\ell}(\sigma, j)=z_{\sigma_{k}}(\sigma, j)=z_{\sigma_{k}}(\sigma, k)+1$, it follows that

$$
\frac{z_{i}(\sigma, j)+1}{s_{i}} \geq \frac{z_{\ell}(\sigma, j)}{s_{\ell}}
$$

for all machines $i$ and $\ell$.

Now let $\sigma$ be a schedule that satisfies (3) and suppose it does not satisfy (22). Then there exist a job $j \in N$ and a machine $i \in M$ such that

$$
\frac{z_{\sigma_{j}}(\sigma, j)+1}{s_{\sigma_{j}}}>\frac{z_{i}(\sigma, j)+1}{s_{i}} .
$$

However, then we have for job $j-1$ that

$$
\frac{z_{\sigma_{j}}(\sigma, j-1)}{s_{\sigma_{j}}}=\frac{z_{\sigma_{j}}(\sigma, j)+1}{s_{\sigma_{j}}}>\frac{z_{i}(\sigma, j)+1}{s_{i}}=\frac{z_{i}(\sigma, j-1)+1}{s_{i}},
$$

which contradicts (3).

An intuitive interpretation for (3) is that, when applying the MFT algorithm, a job that is placed on a machine can not get a better position value than the jobs already placed on any other machine. While it is intuitive that this is indeed a necessary condition for the optimal solution, the intuition that it is also sufficient is not that clear. In that sense, it is indeed a nontrivial reformulation of (2).

\footnotetext{
${ }^{2}$ In case of ties in the SPT ordering, there exist multiple optimal schedules, produced by interchanging symmetric jobs, jobs with equal processing times, or symmetric machines, machines with equal speed, in any optimal schedule. In this case, 2 and 3 describe optimal schedules that correspond to one particular SPT ordering and the rest can be obtained by interchanging symmetric jobs. Note that also machine symmetries are accounted for in 22 and 37 . 


\section{Price of Anarchy for the SPT Scheduling Rule}

In this section we provide both an upper and a lower bound on the price of anarchy for the SPT scheduling rule for the related machine scheduling game. For the problem $\mathrm{Q} \| \sum C_{j}$ with SPT as local scheduling rule, a strategy profile $\nu=\left(\nu_{j}, \nu_{-j}\right)$ is a pure Nash equilibrium if and only if for all jobs $j$ and all machines $i$,

$$
\sum_{\substack{k \leq j \\ \nu_{k} \equiv \nu_{j}}} \frac{p_{k}}{s_{\nu_{j}}} \leq \sum_{\substack{k<j \\ \nu_{k}=i}} \frac{p_{k}}{s_{i}}+\frac{p_{j}}{s_{i}} .
$$

It is well known [14] that the Ibarra-Kim algorithm [17] constructs all Nash equilibria depending on the way ties are broken. This is even true for the more general unrelated machine scheduling problem [14, 18. For related machines the algorithm is described in pseudo-code by Algorithm 2. The Ibarra-Kim algorithm was originally designed as

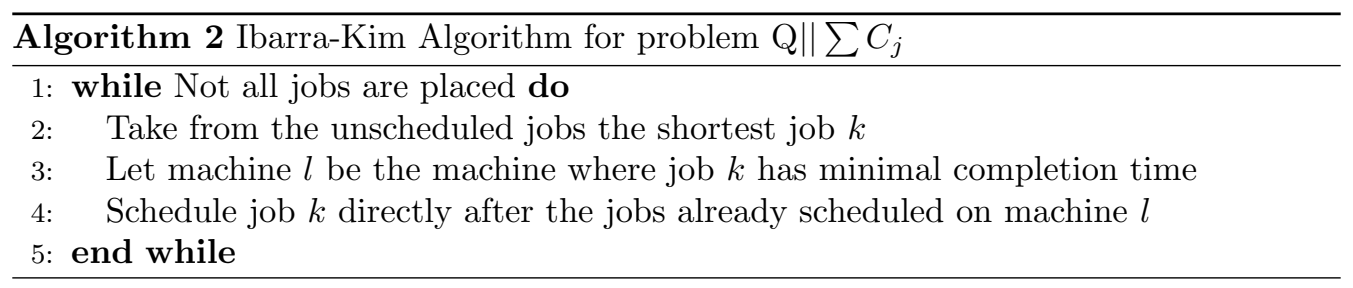

an approximation algorithm for unrelated machine scheduling [17. Therefore, the main result that we discuss in this paper is also an analysis of an upper and lower bound to the performance of this simple greedy algorithm, since the outcomes exactly coincide with Nash equilibria. To the best of our knowledge these performance bounds for the related machine scheduling problem $\mathrm{Q} \| \sum C_{j}$ have not yet been analyzed. Most probably because the problem to find optimal solutions was settled long before 8$]$.

\subsection{Upper bound on the price of anarchy}

Here we establish an upper bound on the price of anarchy for the SPT scheduling rule. Let $\sigma$ be an optimal schedule resulting from the MFT algorithm, and recall that for the objective value in the optimal solution $\sigma$ we have

$$
\sum_{j=1}^{n} C_{j}(\sigma)=\sum_{j=1}^{n}\left(z_{\sigma_{j}}(\sigma, j)+1\right) \frac{p_{j}}{s_{\sigma_{j}}} .
$$

The next theorem is the main result of this paper.

Theorem 3. The price of anarchy for the related machine scheduling problem $\mathrm{Q} \| \sum C_{j}$ with SPT as local sequencing rule is no greater than 2.

Proof. By our previous discussion, it suffices to show that the game is $(2,0)$-semi-smooth, by showing that

$$
\sum_{j=1}^{n} C_{j}\left(\sigma_{j}, \nu_{-j}\right) \leq 2 \sum_{j=1}^{n} C_{j}(\sigma)
$$


for an optimal schedule $\sigma$ and any strategy profile $\nu$.

Let $J_{i}(\sigma)=\left\{j \mid \sigma_{j}=i\right\}$ be the set of jobs scheduled on machine $i$ in the optimal solution $\sigma$. Likewise, let $J_{i}(\nu)=\left\{j \mid \nu_{j}=i\right\}$ be the set of jobs scheduled on machine $i$ in schedule $\nu$. For any job $j$ in $J_{i}(\sigma)$, its completion time $C_{j}\left(\sigma_{j}, \nu_{-j}\right)$ consists of the processing times of all jobs that are on machine $i$ in $\nu$ and that have smaller index than $j$, plus its own processing time on machine $i$. Summing the completion times of all jobs that are on machine $i$ in the optimal solution gives us

$$
\sum_{j \in J_{i}(\sigma)} C_{j}\left(\sigma_{j}, \nu_{-j}\right)=\sum_{j \in J_{i}(\sigma)}\left(\frac{p_{j}}{s_{i}}+\sum_{\substack{k \in J_{i}(\nu) \\ k<j}} \frac{p_{k}}{s_{i}}\right)=\sum_{j \in J_{i}(\sigma)} \frac{p_{j}}{s_{i}}+\sum_{j \in J_{i}(\sigma)} \sum_{\substack{k \in J_{i}(\nu) \\ k<j}} \frac{p_{k}}{s_{i}} .
$$

Note that the number of times that a job $k$ is counted on the right hand side of (5) equals the number of jobs with higher index than $j$ on machine $i$ in the optimal solution, times $1 / s_{i}$. In other words, the second part of (5) can be rewritten as

$$
\sum_{j \in J_{i}(\sigma)} \sum_{\substack{k \in J_{i}(\nu) \\ k<j}} \frac{p_{k}}{s_{i}}=\sum_{k \in J_{i}(\nu)} z_{i}(\sigma, k) \cdot \frac{p_{k}}{s_{i}} .
$$

This gives us

$$
\sum_{j \in J_{i}(\sigma)} C_{j}\left(\sigma_{j}, \nu_{-j}\right)=\sum_{j \in J_{i}(\sigma)} \frac{p_{j}}{s_{i}}+\sum_{k \in J_{i}(\nu)} z_{i}(\sigma, k) \cdot \frac{p_{k}}{s_{i}} .
$$

Now, note that by definition $\sigma_{j}=\nu_{k}=i$, so

$$
\sum_{j \in J_{i}(\sigma)} C_{j}\left(\sigma_{j}, \nu_{-j}\right)=\sum_{j \in J_{i}(\sigma)} \frac{p_{j}}{s_{\sigma_{j}}}+\sum_{k \in J_{i}(\nu)} z_{\nu_{k}}(\sigma, k) \cdot \frac{p_{k}}{s_{\nu_{k}}} .
$$

Summing over all $i$ leads to

$$
\begin{aligned}
\sum_{j=1}^{n} C_{j}\left(\sigma_{j}, \nu_{-j}\right) & =\sum_{i=1}^{m} \sum_{j \in J_{i}(\sigma)} C_{j}\left(\sigma_{j}, \nu_{-j}\right) \\
& =\sum_{i=1}^{m} \sum_{j \in J_{i}(\sigma)} \frac{p_{j}}{s_{\sigma_{j}}}+\sum_{i=1}^{m} \sum_{k \in J_{i}(\nu)} z_{\nu_{k}}(\sigma, k) \cdot \frac{p_{k}}{s_{\nu_{k}}} \\
& =\sum_{j=1}^{n} \frac{p_{j}}{s_{\sigma_{j}}}+\sum_{j=1}^{n} z_{\nu_{j}}(\sigma, j) \cdot \frac{p_{j}}{s_{\nu_{j}}}
\end{aligned}
$$

From Theorem 2 we know

$$
\sum_{j=1}^{n} z_{\nu_{j}}(\sigma, j) \cdot \frac{p_{j}}{s_{\nu_{j}}} \leq \sum_{j=1}^{n}\left(z_{\sigma_{j}}(\sigma, j)+1\right) \cdot \frac{p_{j}}{s_{\sigma_{j}}}=\sum_{j=1}^{n} C_{j}(\sigma) .
$$


Also, the completion time of any job is at least its processing time on the machine it is scheduled on, so

$$
\sum_{j=1}^{n} \frac{p_{j}}{s_{\sigma_{j}}} \leq \sum_{j=1}^{n} C_{j}(\sigma)
$$

Combining the above, we get

$$
\sum_{j=1}^{n} C_{j}\left(\sigma_{j}, \nu_{-j}\right) \leq 2 \sum_{j=1}^{n} C_{j}(\sigma)
$$

for all strategy profiles $\nu$.

\subsection{Lower bound on the price of anarchy}

Here we describe a parametric instance which has price of anarchy approaching $e /(e-1)$ as the number of machines, jobs, and the maximum speed of the fastest machine grows. The Nash equilibrium will be the schedule with all jobs on the fastest machine.

Instance 1. Let $\mathcal{I}$ be the set of parametric instances $I(s, m)$ that satisfy the following. $I(s, m)$ has $m$ machines, one with speed $s \in \mathbb{N}$, with $s>1$, and all the other machines with speed 1 . We assume that $m>s$. Furthermore, $I(s, m)$ has $n=m-1+s$ jobs, with processing requirements

$$
p_{j}= \begin{cases}1 & \text { if } 1 \leq j \leq s-1 \\ x^{j-s} & \text { if } s \leq j \leq n\end{cases}
$$

where $x:=s /(s-1)$.

Lemma 4. Instances from $\mathcal{I}$ have a Nash equilibrium with all jobs on the fastest machine.

Proof. In the schedule with all jobs in SPT order on the fastest machine, the completion time of a job $j<s$ is equal to

$$
C_{j}=\sum_{k=1}^{j} \frac{1}{s}=\frac{j}{s}<1 .
$$

Observe that $1 /(x-1)=s-1$. Therefore, for job $j \geq s$, the completion time is equal to

$$
\begin{aligned}
C_{j}=\sum_{k=1}^{j} \frac{p_{k}}{s} & =\frac{1}{s}\left((s-1)+\sum_{k=0}^{j-s} x^{k}\right) \\
& =\frac{1}{s}\left((s-1)+\frac{x^{j-s+1}-1}{x-1}\right) \\
& =\frac{1}{s}\left((s-1)+(s-1) x^{j-s+1}-(s-1)\right) \\
& =x^{j-s}=p_{j} .
\end{aligned}
$$

Thus, the Nash equilibrium condition (4) holds for all jobs, since all other machines have speed 1. 
We use this to compute a lower bound on the price of anarchy.

Theorem 5. The (pure strategy) price of anarchy for the related machine scheduling problem $\mathrm{Q} \| \sum C_{j}$ with $S P T$ local scheduling rule is at least e/ $(e-1)$.

Proof. Consider instances $I(s, m)$ from $\mathcal{I}$ as defined above. Consider the (optimal) assignment where the $s$ longest jobs $n-s+1=m, \ldots, n$ are on the fast machine, while the first $n-s=m-1$ jobs are each on a slow machine. Note that, because $m>s$ also jobs with $p_{j}>1$ will be scheduled on slow machines (jobs $s, \ldots, m-1$ ). The objective value in this solution is therefore equal to

$$
\begin{aligned}
\sum_{j=1}^{s-1} 1+\sum_{j=s}^{m-1} x^{j-s}+\frac{1}{s} \sum_{j=m}^{n} \sum_{k=m}^{j} x^{k-s} & =(s-1)+\sum_{j=0}^{m-s-1} x^{j}+\frac{1}{s} \sum_{j=m}^{n}\left(\sum_{k=0}^{j-s} x^{k}-\sum_{k=0}^{m-s-1} x^{k}\right) \\
& =(s-1)+(s-1)\left(x^{m-s}-1\right)+\sum_{j=m}^{n}\left(x^{j-s}-x^{m-s-1}\right) \\
& =(s-1) x^{m-s}+\sum_{j=m-s}^{n-s} x^{j}-\sum_{j=m}^{n} x^{m-s-1} \\
& =s x^{m-s-1}+(s-1)\left(x^{n-s+1}-x^{m-s}\right)-s x^{m-s-1} \\
& =(s-1)\left(x^{n-s+1}-x^{m-s}\right) .
\end{aligned}
$$

To compute the objective value for the Nash equilibrium, recall Lemma 4 and the expressions for the job completion times in (8) and (9). From this we compute the objective value in the Nash equilibrium as

$$
\begin{aligned}
\sum_{j=1}^{s-1} \frac{j}{s}+\sum_{j=s}^{n} x^{j-s} & =\frac{(s-1)}{2}+\sum_{j=0}^{n-s} x^{j} \\
& =\frac{(s-1)}{2}+(s-1)\left(x^{n-s+1}-1\right) \\
& =(s-1)\left(x^{n-s+1}-\frac{1}{2}\right) .
\end{aligned}
$$

Combining (10) and 111) gives us the following bound for the price of anarchy

$$
\operatorname{PoA}(I(s, m)) \geq \frac{x^{n-s+1}-\frac{1}{2}}{x^{n-s+1}-x^{m-s}}=\frac{x^{s}-\frac{1}{2} x^{-(m-s)}}{x^{s}-1} .
$$

Now if we let $m$ (and $n$ ) go to infinity, and because $x>1$, we get that

$$
\lim _{m \rightarrow \infty} \operatorname{PoA}(I(s, m)) \geq \frac{x^{s}}{x^{s}-1}=\frac{\left(\frac{s}{s-1}\right)^{s}}{\left(\frac{s}{s-1}\right)^{s}-1} .
$$

Now, the right hand side of 12 converges to $e /(e-1)$ as $s$ goes to infinity. 


\section{Identical Machines}

Two special cases arise when either the machines or the jobs are all identical. In both cases, note that all pure Nash equilibria are optimal solutions, and the (pure) price of anarchy would equal 1. However, even if both the machines and the jobs are identical, i.e., $s_{i}=1$ for all machines $i$ and $p_{j}=1$ for all jobs $j$, mixed Nash equilibria maye have price of anarchy $\approx 3 / 2$.

Theorem 6 (Folklore). The (mixed strategy) price of anarchy for the parallel machine scheduling problem $\mathrm{P} \| \sum C_{j}$ with SPT local scheduling rule is at least $3 / 2-1 /(2 m)$.

Proof. Consider an instance with $n$ jobs and $m=n$ machines. The optimal solution has one job on each machine and $\sum_{j} C_{j}=n$. Assume that ties are broken according to index. Now consider the mixed Nash equilibrium $\nu$, where each job is scheduled on each machine with probability $1 / n$. This is a Nash equilibrium, as the expected load of the jobs with index less than $j$ is divided equally over all machines. Now, for any job $j$, the expected completion time is equal to

$$
\mathbb{E}_{\nu} C_{j}(\nu)=1+\frac{j-1}{n}
$$

Summing over all jobs gives

$$
\sum_{j=1}^{n} \mathbb{E}_{\nu} C_{j}(\nu)=\sum_{j=1}^{n} 1+\frac{j-1}{n}=n+\frac{n(n-1)}{2} n=\frac{3 n}{2}-\frac{1}{2} .
$$

Dividing by $n=m$ gives $3 / 2-1 /(2 m)$.

The identical machine model, where all machines have speed 1 , has robust price of anarchy of exactly $3 / 2-1 /(2 m)$. This result was also found by Rivera 24] and Rahn and Schäfer 23. Here we give a short and simple proof that we discussed in private communication with J.R. Correa. The proof follows the framework of $\beta$-niceness as defined by Augustine et al. 2 and its extended version, $(\lambda, \mu)$-niceness as defined by Anshelevich et al. [1. In fact, we prove that the game is $\left(\frac{3}{2}-\frac{1}{2 m}\right)$-nice and thus also $\left(\frac{3}{2}-\frac{1}{2 m}, 0\right)$-nice. This implies bounds on Nash equilibria, mixed Nash equilibria and correlated equilibria, but not coarse correlated equilibria [1].

Theorem 7. The price of anarchy for the parallel machine scheduling problem $\mathrm{P} \| \sum C_{j}$ with SPT local scheduling rule is $3 / 2-1 /(2 m)$.

Proof. We are only left to prove the upper bound. Again, let the jobs be indexed according to the order in which the machines process them. For any strategy profile $\nu$ and any job $j$, let $\nu_{j}^{\prime}$ be a best response of $j$ to $\nu_{-j}$, so that

$$
C_{j}\left(\nu_{j}^{\prime}, \nu_{-j}\right) \leq C_{j}\left(\nu_{j}^{*}, \nu_{-j}\right) .
$$

As any job $j$ has to care only about the jobs $k<j$, by a standard averaging argument over the machines, irrespective of $\nu_{-j}$ there must be a machine where job $j$ can get a start time at most $\sum_{k<j} p_{k} / m$. Since this holds for all jobs $j \in N$, we have

$$
\sum_{j \in J} C_{j}\left(\nu_{j}^{\prime}, \nu_{-j}\right) \leq \sum_{\substack{j \in J \\ 10}} \sum_{k<j} \frac{p_{k}}{m}+\sum_{j \in J} p_{j}
$$


Now let $\sigma$ be a strategy profile that results in an optimal solution. The lower bound on the optimal solution from Eastman et al. [12, Thm. 1] gives:

$$
\sum_{j \in J} \sum_{k=1}^{j} \frac{p_{k}}{m}+\left(\frac{1}{2}-\frac{1}{2 m}\right) \sum_{j \in J} p_{j} \leq \sum_{j \in J} C_{j}(\sigma)
$$

Therefore

$$
\begin{aligned}
\sum_{j \in J} C_{j}\left(\nu_{j}^{\prime}, \nu_{-j}\right) & \leq \sum_{j \in J} \sum_{k=1}^{j-1} \frac{p_{k}}{m}+\sum_{j \in J} p_{j} \\
& \leq \sum_{j \in J} \sum_{k=1}^{j} \frac{p_{k}}{m}+\left(1-\frac{1}{m}\right) \sum_{j \in J} p_{j} \\
& \leq \sum_{j \in J} C_{j}(\sigma)+\left(\frac{1}{2}-\frac{1}{2 m}\right) \sum_{j \in J} p_{j} \\
& \leq\left(\frac{3}{2}-\frac{1}{2 m}\right) \sum_{j \in J} C_{j}(\sigma) .
\end{aligned}
$$

This proves that the game is $\left(\frac{3}{2}-\frac{1}{2 m}, 0\right)$-nice and therefore the price of anarchy is at most $3 / 2-1 /(2 m)$.

\section{Concluding Remarks}

We leave open to determine the exact value for the price of anarchy in $[1.58,2]$. The gap may be due to the fact that our proof for the upper bound yields a robust price of anarchy bound, while for the parametric instances from Theorem 5 scheduling any job on the fastest machine is even a pure strategy dominant strategy equilibrium.

This leaves open the possibility that 2 is the exact value for the robust price of anarchy, while the exact value for pure strategy Nash equilibria is $e /(e-1)$. We believe that an improvement of the upper bound of 2 is possible, however. This because either of the terms that appear in our analysis in (6) and (7) can be equal to the optimum value, but we have not been able to construct instances where both inequalities are tight simultaneously.

\section{References}

[1] E. Anshelevich, J. Postl, and T. Wexler. Assignment games with conflicts: Robust price of anarchy and convergence results via semi-smoothness. Theory of Computing Systems, 59(3):440-475, 2016. doi: $10.1007 / \mathrm{s} 00224-015-9646-0$.

[2] J. Augustine, N. Chen, E. Elkind, A. Fanelli, N. Gravin, and D. Shiryaev. Dynamics of profit-sharing games. Internet Mathematics, 11(1):1-22, 2015. doi: 10.1080/15427951.2013.830164.

[3] Y. Azar, L. Fleischer, K. Jain, V. Mirrokni, and Z. Svitkina. Optimal coordination mechanisms for unrelated machine scheduling. Operations Research, 63(3):489-500, 2015. doi: 10.1287/opre.2015. 1363.

[4] S. Bhattacharya, S. Im, J. Kulkarni, and K. Munagala. Coordination mechanisms from (almost) all scheduling policies. In Proceedings of the 5th Conference on Innovations in Theoretical Computer Science, pages 121-134. ACM, 2014. doi: 10.1145/2554797.2554811. 
[5] I. Caragiannis, C. Kaklamansis, P. Kanellopoulos, M. Kyropoulou, B. Lucier, R. Paes Leme, and É. Tardos. Bounding the inefficiency of outcomes in generalized second price auctions. Journal of Economic Theory, 156:343-388, 2015. doi: 10.1016/j.jet.2014.04.010.

[6] G. Christodoulou, E. Koutsoupias, and A. Nanavati. Coordination mechanisms. Theoretical Computer Science, 410(36):3327 - 3336, 2009. doi: 10.1016/j.tcs.2009.01.005.

[7] R. Cole, J. R. Correa, V. Gkatzelis, V. Mirrokni, and N. Olver. Decentralized utilitarian mechanisms for scheduling games. Games and Economic Behavior, 92:306-326, 2015. doi: 10.1016/j.geb.2013. 03.011 .

[8] R. W. Conway, W. L. Maxwell, and L. W. Miller. Theory of scheduling. Addison-Wesley Publishing Co., 1967.

[9] J. R. Correa and M. Queyranne. Efficiency of equilibria in restricted uniform machine scheduling with total weighted completion time as social cost. Naval Research Logistics, 59(5):384-395, 2012. doi: $10.1002 /$ nav.21497.

[10] A. Czumaj and B. Vöcking. Tight bounds for worst-case equilibria. ACM Trans. Algorithms, 3(1): 4:1-4:17, Feb. 2007. doi: 10.1145/1186810.1186814.

[11] P. Dubey. Inefficiency of Nash equilibria. Mathematics of Operations Research, 11(1):1-8, 1986. doi: $10.1287 /$ moor.11.1.1.

[12] W. L. Eastman, S. Even, and I. M. Isaacs. Bounds for the optimal scheduling of $n$ jobs on $m$ processors. Management Science, 11(2):268-279, 1964. doi: 10.1287/mnsc.11.2.268.

[13] R. Graham, E. Lawler, J. Lenstra, and A. Rinnooy Kan. Optimization and approximation in deterministic sequencing and scheduling: a survey. Annals of Discrete Mathematics, 5:287-326, 1979. doi: 10.1016/S0167-5060(08)70356-X.

[14] B. Heydenreich, R. Müller, and M. Uetz. Games and mechanism design in machine scheduling an introduction. Production and Operations Management, 16(4):437-454, 2007. doi: 10.1111/j. 1937-5956.2007.tb00271.x.

[15] R. Hoeksma and M. Uetz. The price of anarchy for minsum related machine scheduling. In R. SolisOba and G. Persiano, editors, Approximation and Online Algorithms, volume 7164 of Lecture Notes in Computer Science, pages 261-273. Springer, 2012. doi: 10.1007/978-3-642-29116-6_22.

[16] E. Horowitz and S. Sahni. Exact and approximate algorithms for scheduling nonidentical processors. Journal of the ACM, 23(2):317-327, Apr. 1976. doi: 10.1145/321941.321951.

[17] O. H. Ibarra and C. E. Kim. Heuristic algorithms for scheduling independent tasks on nonidentical processors. Journal of the ACM, 24(2):280-289, 1977. doi: 10.1145/322003.322011.

[18] N. Immorlica, L. E. Li, V. S. Mirrokni, and A. S. Schulz. Coordination mechanisms for selfish scheduling. Theoretical Computer Science, 410(17):1589-1598, 2009. doi: 10.1016/j.tcs.2008.12.032

[19] E. Koutsoupias and C. Papadimitriou. Worst-case equilibria. In C. Meinel and S. Tison, editors, 16th Annual Symposium on Theoretical Aspects of Computer Science, volume 1563 of Lecture Notes in Computer Science, pages 404-413. Springer, 1999. doi: 10.1007/3-540-49116-3_38.

[20] B. Lucier and R. Paes Leme. Gsp auctions with correlated types. In Proceedings of the 12th ACM Conference on Electronic Commerce, EC '11, pages 71-80, New York, NY, USA, 2011. ACM. doi: 10.1145/1993574.1993587.

[21] R. B. Myerson. Utilitarianism, egalitarianism, and the timing effect in social choice problems. Econometrica: Journal of the Econometric Society, pages 883-897, 1981. doi: 10.2307/1912508.

[22] C. Papadimitriou. Algorithms, games, and the internet. In Proceedings of the Thirty-third Annual ACM Symposium on Theory of Computing, pages 749-753. ACM, 2001. doi: 10.1145/380752. 380883.

[23] M. Rahn and G. Schäfer. Bounding the inefficiency of altruism through social contribution games. In Y. Chen and N. Immorlica, editors, Web and Internet Economics, volume 8289 of Lecture Notes in Computer Science, pages 391-404. Springer, 2013. doi: 10.1007/978-3-642-45046-4_32.

[24] O. L. Rivera. Cotas para el precio de la anarquía de juegos de scheduling. Master's thesis, Universidad de Chile, 2012. URL http://www.repositorio.uchile.cl/handle/2250/111965

[25] T. Roughgarden. Intrinsic robustness of the price of anarchy. J. ACM, 62(5):32:1-32:42, Nov. 2015. doi: $10.1145 / 2806883$.

[26] L. Yu, K. She, H. Gong, and C. Yu. Price of anarchy in parallel processing. Information Processing Letters, 110(8-9):288-293, 2010. doi: 10.1016/j.ipl.2010.02.003. 